\title{
Very-Large-Eddy Simulation of Nonreactive Turbulent Flow for Annular Trapped Vortex Combustor
}

\author{
K. Zhang, Y. Jin ${ }^{\dagger}$, X. Han and X. He \\ College of Energy and Power Engineering, Nanjing University of Aeronautics and Astronautics, Nanjing, \\ Jiangsu, 210016, China \\ †Corresponding Author Email: pde_jy@nuaa.edu.cn
}

(Received July 13, 2021; accepted November 5, 2021)

\begin{abstract}
A hybrid Reynolds-averaged Navier-Stokes (RANS) large eddy simulation (LES) method is applied in this work. It called very-large-eddy simulation (VLES) turbulence closure model. The aim of this present study is firstly to validate the accuracy of this method for a specific engineering application (a trapped vortex combustor) and secondly to describe its flow characteristics. The trapped vortex combustor is a new concept that utilizes a large recirculation vortex to stabilize the flame. An accurate prediction of the turbulent flow is meaningful for the trapped vortex combustor. The time-averaged velocity, root-mean-square (rms) velocity, and flow pattern are compared with the experimental data. And the LES model, RANS BSL $k$ - $\omega$ model, and RANS $k$ - $\varepsilon$ model are also applied for the simulation with different mesh resolutions. The results show that the VLES BSL $k-\omega$ model provides improved accuracy for velocity prediction. The classical large vortex structure for the trapped vortex combustor is captured qualitatively by the VLES BSL $k-\omega$ model also. In addition, the vortex breakdown and processing vortex cone are visualized using the Q-criterion. Furthermore, the VLES BSL $k$ - $\omega$ model is not sensitive to the gird resolution. The VLES method is able to predict the turbulent flow of trapped vortex combustor relatively well.
\end{abstract}

Keywords: Hybrid RANS-LES method; Very-large-eddy simulation; Trapped vortex Combustor flow.

\section{INTRODUCTION}

In many industrial and engineering applications, such as aero engines, the Reynolds-averaged NavierStokes (RANS) model is still the dominant method for simulating internal flows with high turbulence intensity. However, the industry meets the new requirement of dealing with the instantaneous flow aerodynamics, which the RANS models cannot solve very well. The large eddy simulation (LES) method is a candidate which large eddies are computed, and small-eddies (subgrid-scale) are modeled. However, it is not widely used in industrial simulations owing it very restrictive mesh resolution and overrestrained near-wall treatment for accuracy (Sagaut 2006). The computational cost is high for high Reynolds number flows in engineering aerodynamics (Sagaut et al. 2012).

In the past two decades, the new hybrid RANS-LES method has become increasingly popular and has shown potential in some applications (Langhe et al. 2005; Davidson and Dahlström 2005). This method combines the advantages of the two different models. The RANS models act on the near-wall region, and the LES models act on the region far from the boundary layer flows.
The detached eddy simulation (DES) is one of the hybrid methods, and it has been used for many turbulent flows (Spalart 2008). However, the feature of the 'weak' RANS-LES coupling method impedes its development. The 'weak' RANS-LES coupling method means that the empirical connection interface between the RANS and LES regions, in other words, there is no specific transformation mechanism between the modeled turbulence energy and the resolved turbulence energy. This leads to limited application of the method in complex turbulence flows for industrial simulation. stressblended eddy simulation (SBES) is developed by Menter (2018), which is also a popular hybrid RANS-LES model. SBES is a fairly simple concept where existing RANS and LES models are 'blended' by a shielding (or blending) function. The SBES turbulence model and the flamelet generated manifold (FGM) combustion model are both used by Xia et al. (2020). The results have been shown to be capable of accurately modeling the film-cooled scenarios.

Speziale (1998) first proposed the concept of very-large-eddy simulation (VLES) firstly as a self-adaptive hybrid method; it is later called the flow simulation methodology (Fasel et al. 2002). 
Following this idea, there are other hybrid methods similar to VLES (Batten et al. 2004; Hsieh et al. 2009; Labois and Lakehal 2011; Liu and Shih 2006). However, Speziale's VLES model damps the Reynolds stress excessively, and it is tough for the original Speziale's VLES model to recover the RANS method unless the mesh is unreasonably coarse (Zhang et al. 2000). Although the original VLES model can reach both direct numerical simulation (DNS) and RANS limits, a correct LES model is not worked specifically.

Hence, a new VLES model was proposed to resolve the problems of the original Speziale's VLES model. The improved VLES method has been validated using several turbulent flows, such as the turbulent channel flow at $\mathrm{Re}=395$ (Han and Krajnović 2012a; Han et al. 2012b), turbulent flow past a square cylinder at $\mathrm{Re}=22000$ (Han and Krajnović 2012; Han et al. 2012 ) and $\mathrm{Re}=3000$ (Han and Krajnović 2013a), turbulent flow past a circular cylinder at $\mathrm{Re}=3900$ (Han and Krajnović 2015), $\mathrm{Re}=140000$ (Han and Krajnović 2013a), and Re=3.0E+06 (Han and Krajnović 2015), backward-facing step flow at $\mathrm{Re}=40000$ (Han and Krajnović 2013a), turbulent flow past a D-shaped cylinder (Han and Krajnović 2013b), and periodic hill flow at $\mathrm{Re}=10595$ (Han and Krajnović 2012a). The improved VLES model shows fine accuracy prediction of mean and rms velocity. Recently, a strong swirling flow, $\mathrm{Sr}=1.16$ and $\mathrm{Sr}=1.23$, has also been simulated using the VLES model. The study confirms the validity and accuracy of the VLES model for complex strongly swirling turbulent flow (Xia et al. 2020; Tiwari et al. 2020).

It should be noted that the previous validations of the VLES model were performed with some classic turbulent flows; however, industrial turbulent flow validation is insufficient. The purpose of the present work is to operate the improved VLES model work on the trapped vortex combustor (TVC) turbulent flow to validate its accuracy. Hsu et al. (1995) proposed the TVC concept. Instead of swirlstabilization, TVC uses the cavity to stabilize the flame. If the cavity geometry is carefully designed, the large-scale vortex is trapped in the cavity region and is less disturbed by the unstable mainstream flow. The RANS models have been widely used in TVC simulations. Jin et al. (2014a) and Jin et al. (2014b) used the $k-\varepsilon$ model to simulate the cold flow of TVC.

Chen and Zhao (2018) studied a trapped vortex ramjet combustor using the Reynolds stress model (RSM). The LES model has been used by Merlin et al. (2012) and Sharifzadeh and Afshari (2020). For TVC and VLES models, it is meaningful to simulate the turbulent flows of the TVC using the new improved VLES model. Thus, in the present study, the VLES turbulence model is applied based on the RANS BSL $k-\omega$ model framework to predict the flow in a TVC. The computational results obtained from the VLES BSL $k-\omega$ model are compared with experimental data available from previous work (Burguburu 2012). The flow characteristics are also discussed in this work.

\section{Numerical Details}

\subsection{Mean Flow Equations}

In the present VLES framework, the Navier-Stokes equations for incompressible Newtonian fluids are solved, they are expressed as

$\frac{\partial \rho}{\partial t}+\frac{\partial}{\partial x_{j}}\left(\rho U_{j}\right)=0$

$\frac{\partial}{\partial t}\left(\rho U_{i}\right)+\frac{\partial}{\partial x_{j}}\left(\rho U_{i} U_{j}\right)=-\frac{\partial p}{\partial x_{i}}$

$+\frac{\partial}{\partial x_{j}}\left[\left(\mu+\mu_{t}\right)\left(\frac{\partial U_{i}}{\partial x_{j}}+\frac{\partial U_{j}}{\partial x_{i}}\right)\right]$

where $\rho$ is the fluid density, $U_{i}$ is the velocity component, $p$ is the pressure, $\mu$ is the laminar viscosity, and $\mu_{t}$ is the turbulent viscosity.

\subsection{VLES Modeling}

In the VLES $k-\omega$ modeling, the transport equations for the modeled turbulence kinetic energy, $k$, and its dissipation rate, $\omega$, are exactly the same as the BSL $k-\omega$, given by

$$
\begin{aligned}
& \frac{D \rho k}{D t}=P_{k}-\beta^{*} \rho k \omega+\frac{\partial}{\partial x_{j}}\left[\left(\mu+\sigma_{k 1} \mu_{t}\right) \frac{\partial k}{\partial x_{j}}\right] \\
& \frac{D \rho \omega}{D t}=\omega\left(\gamma_{1} \frac{P_{k}}{k}-\beta_{1} \rho \omega\right)+\frac{\partial}{\partial x_{j}}\left[\left(\mu+\sigma_{\omega 1} \mu_{t}\right) \frac{\partial \omega}{\partial x_{j}}\right] \\
& +2 \rho\left(1-F_{1}\right) \sigma_{\omega 2} \frac{1}{\omega} \frac{\partial k}{\partial x_{j}} \frac{\partial \omega}{\partial x_{j}}
\end{aligned}
$$

where $P_{k}$ is the kinetic energy production in the form of

$$
P_{k}=2 \mu_{t} S_{i j} S_{i j}
$$

with $S_{i j}$ being the strain rate tensor:

$S_{i j}=\frac{1}{2}\left(\frac{\partial U_{i}}{\partial x_{j}}+\frac{\partial U_{j}}{\partial x_{i}}\right)$

The form of the RANS modeling is not changed from that of the BSL $k-\omega$ model. The VLES $k-\omega$ model only modifies the formulation of the turbulent viscosity $\mu_{t}$ in the equation

$\mu_{t}=F r \cdot \rho k / \omega$

Similarly, the VLES $k-\varepsilon$ modeling is

$$
\begin{aligned}
& \frac{D \rho k}{D t}=P_{k}-\rho \varepsilon+\frac{\partial}{\partial x_{j}}\left[\left(\mu+\frac{\mu_{t}}{\sigma_{k}}\right) \frac{\partial k}{\partial x_{j}}\right] \\
& \frac{D \rho \varepsilon}{D t}=\frac{\varepsilon}{k}\left(C_{\varepsilon 1} P_{k}-C_{\varepsilon 2} \rho \varepsilon\right)+\frac{\partial}{\partial x_{j}}\left[\left(\mu+\frac{\mu_{t}}{\sigma_{\varepsilon}}\right) \frac{\partial \varepsilon}{\partial x_{j}}\right]
\end{aligned}
$$

$\mu_{t}=F r \cdot \rho C_{\mu} k^{2} / \varepsilon$ 
The key parameter $F r$ is the core of the present VLES modeling. The form is

$$
F r=\min \left[1.0,\left(\frac{1.0-\exp \left(-\beta L_{c} / L_{k}\right)}{1.0-\exp \left(-\beta L_{i} / L_{k}\right)}\right)^{2}\right]
$$

where the function $\min (x, y)$ refers to the minimum value between $x$ and $y, \beta$, and $n$ are the model constants. The parameters $L_{c}, L_{i}$, and $L_{k}$ are the cut-off length scale, integral length scale, and Kolmogorov length scale, respectively, defined as

$$
\begin{aligned}
& L_{c}=C_{x}\left(\Delta_{x} \Delta_{y} \Delta_{z}\right)^{1 / 3} \\
& L_{i}=k^{3 / 2} / \varepsilon ; \quad L_{k}=v^{3 / 4} / \varepsilon^{1 / 4}
\end{aligned}
$$

where $c_{x}$ has been discussed in a previous VLES modeling (Han and Krajnović (2015)); $\Delta_{x}, \Delta_{y}$ and $\Delta_{z}$ are mesh scales in different directions; and $v$ is the laminar viscosity. The model constant $C_{x}$ is identified following the idea that the standard $k-\omega$ model is identical to the Smagorinsky-type LES model when $L_{c}=L_{i}$. It is related to the formulation as:
$C_{x}=\sqrt{0.3} C_{s} / \beta^{*}$

$C_{s}=\max \left[\sqrt{\frac{\left[\left(C_{S, 0}^{2} \Delta^{2}|S|\right)^{2}+v^{2}\right]^{1 / 2}-v}{\Delta^{2}|S|}}, 0.002\right]$

where the function $\max (x, y)$ refers to the maximum value between $x$ and $y$, and $C_{s, 0}=0.1$ is the Smagorinsky model constant.

Table 1 Model constants for the VLES BSL $k$ - $\omega$ turbulence model.

\begin{tabular}{|c|c|c|c|}
\hline $\begin{array}{c}\text { Model } \\
\text { contant }\end{array}$ & Value & Model constant & Value \\
\hline$\beta$ & 0.002 & $\sigma_{k 1}$ & 0.5 \\
\hline$C_{\mu}$ & 0.09 & $\sigma_{\omega 1}$ & 0.5 \\
\hline$\sigma_{k}$ & 1.0 & $\beta_{1}$ & 0.0750 \\
\hline$\sigma_{\varepsilon}$ & 1.3 & $\gamma_{1}$ & 0.553 \\
\hline$C_{\varepsilon 1}$ & 1.44 & $\sigma_{k 2}$ & 1.0 \\
\hline$C_{\varepsilon 2}$ & 1.92 & $\sigma_{\omega 2}$ & 0.856 \\
\hline$\beta^{*}$ & 0.09 & $\beta_{2}$ & 0.0828 \\
\hline$n$ & 2 & $\gamma_{2}$ & 0.440 \\
\hline
\end{tabular}

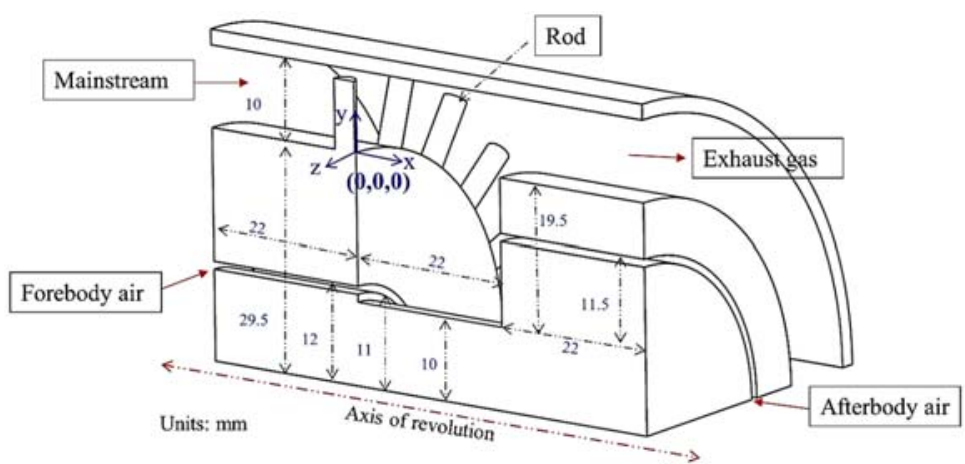

Fig. 1. Sector view of $1 / 4$ of the annular TVC.

The resolution control function $F r$ has a more concrete physical meaning in the present VLES model, which approximately resembles the ratio of the unresolved turbulent kinetic energy to the total turbulent kinetic energy. It can be seen that the control function $F r$ has a value between 0 and 1, which determines how much of the turbulence is modeled. For example, RANS behavior modeling will be recovered when $F r$ approaches 1; LESbehavior modeling will be recovered when $F r<1$; and the DES limit for the VLES will approach when $\mathrm{Fr}$ approaches 0 . This feature is similar to the existing DES hybrid method; however, the attached boundary layer is not solved entirely in the VLES modeling compared with the DES method.

\subsection{Numerical Method}

The VLES modeling is performed in a general CFD code by use of the finite-volume method. And the convective terms are achieved using a bounded second-order central differencing scheme. The second-order upwind scheme is adopted for turbulence model equations, and the temporal discretization is discretized using a second-order implicit scheme. Finally, the SIMPLEC algorithm is applied for pressure-velocity coupling. The velocityinlet and pressure-outlet boundary conditions are employed at the inlet and outlet for all models. And the no-slip condition is applied at the solid walls. The synthetic turbulence generator is adopted for the VLES and LES air inlets. For the LES model, the wall-adapting local eddy-viscosity (WALE) solution is used for the subgrid-scale model. For the RANS model, stand wall functions are used in numerical simulations for the near-wall region. The flow fields are statistically time averaged over approximately ten flow characteristics times after five flow characteristics times.

\section{Computational Details}

A turbulent flow through an annular TVC has been investigated, with reference to experiments (Burguburu 2012; Merlin et al. 2012). Figure 1 


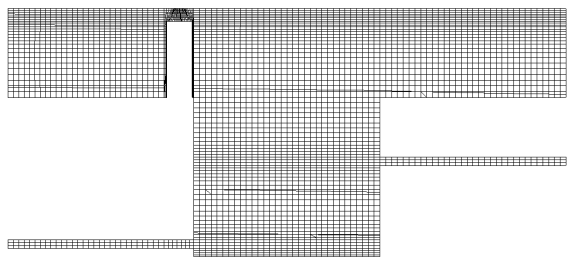

(a) gird 1 (M1)

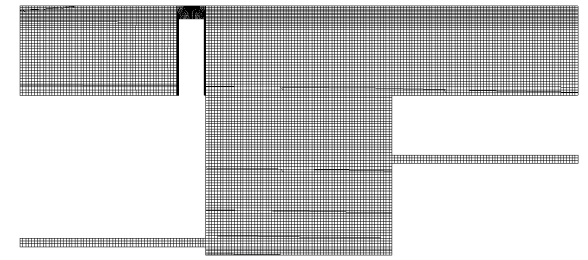

(b) grid 2 (M2)

Fig. 2. Grid topology of the annular TVC.

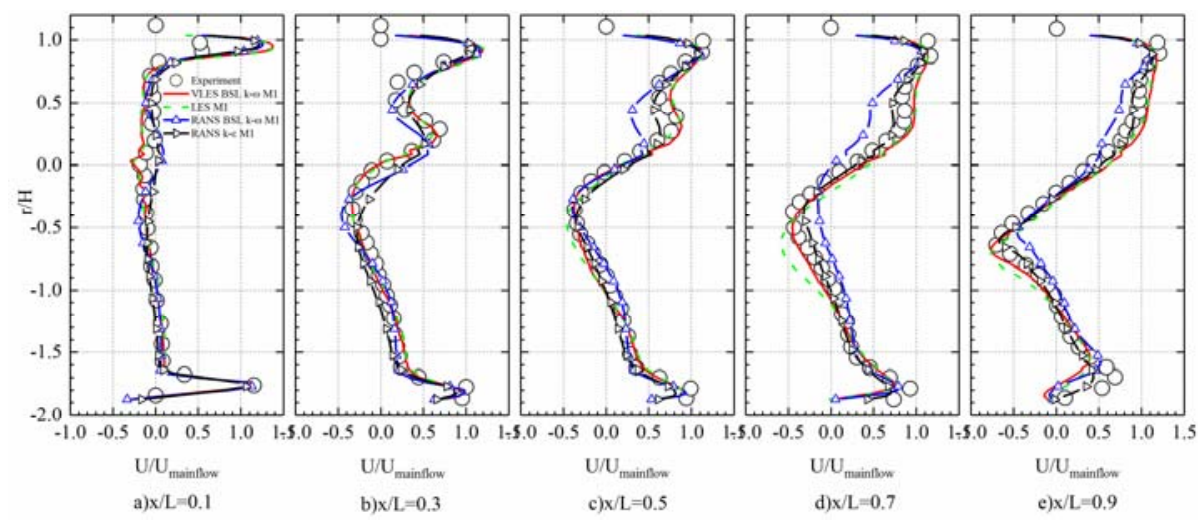

Fig. 3. Radial distributions of $x$ mean velocity $(U)$ at plane crossing rods, comparison of experimental data, VLES BSL k- $\omega$ model, LES model, RANS BSL k- $\omega$ model, RANS k- $\varepsilon$ model, on grid M1.

shows the sector view of one quarter of the annular TVC. The depth and length of the cavity are 19.5 and $22 \mathrm{~mm}$, respectively. The distance from the cavity forebody slot to the axis of revolution is $12 \mathrm{~mm}$, whereas that from the cavity afterbody slot is 22.5 $\mathrm{mm}$ and the width of each slot is $1 \mathrm{~mm}$. The height of the mainstream channel is $10 \mathrm{~mm}$. The flow is specified to be along the $\mathrm{x}$-direction, and the radial direction of the model is the $y$-direction. The flame holder facility section consists of 20 rods with a diameter of $3 \mathrm{~mm}$, uniformly distributed around the forebody along the path of the mainstream air flow.

Figure 2 shows the grid topology of the annular TVC. Two grid resolutions are adopted to examine the accuracy of the new VLES model. The coarse grid M1 is composed of approximately 239 million hexahedral cells, and the grid resolutions in the Cartesian directions are $0.08 \mathrm{~mm}<\Delta \mathrm{x}<0.68 \mathrm{~mm}$ and $0.12 \mathrm{~mm}<\Delta \mathrm{y}=\Delta \mathrm{z}<0.68 \mathrm{~mm}$. The relatively fine grid M2 comprises approximately 884 million hexahedral cells, and the grid resolutions in the Cartesian directions are $0.05 \mathrm{~mm}<\Delta \mathrm{x}<0.3 \mathrm{~mm}$ and $0.16 \mathrm{~mm}<\Delta \mathrm{y}=\Delta \mathrm{z}<0.33 \mathrm{~mm}$. Three air inlet conditions for simulation are listed in Table 2.

Table 2 Three air inlet conditions for simulation (Merlin et al. 2012).

\begin{tabular}{|c|c|c|c|}
\hline Injection & $\operatorname{Air}(\mathrm{g} / \mathrm{s})$ & $\begin{array}{c}\text { Velocity } \\
(\mathrm{m} / \mathrm{s})\end{array}$ & Reynolds \\
\hline Mainstream & 18 & 7.0 & 3500 \\
\hline $\begin{array}{c}\text { Forebody } \\
\text { air }\end{array}$ & 0.7 & 7.8 & 390 \\
\hline $\begin{array}{c}\text { Afterbody } \\
\text { air }\end{array}$ & 1 & 6.1 & 300 \\
\hline
\end{tabular}

\section{RESULTS AND DISCUSSION}

\subsection{The Mean and Rms Velocity Profile}

In this work, the velocity and radial distance are normalized by the mainstream mean velocity, $U_{\text {mainflow }}$, and the mainstream channel height, $H$. The normalized mean $\mathrm{x}$ velocity and rms $\mathrm{x}$ velocity are compared with the experimental measurements for two different planes. One is a plane crossing the rods $(\mathrm{x} / \mathrm{L}=0.1,0.3,0.5,0.7$, and 0.9 , where $L$ represents the length of the cavity), and the other is plane between the rods $(\mathrm{x} / \mathrm{L}=0.1,0.2,0.3,0.4$, and 0.5$)$.

Figure 3 shows the mean $x$ velocity obtained by the VLES, LES, and RANS models on a coarse mesh (M1) compared with the experimental results. It can be seen that the agreement between the VLES predictions and experimental results is significant; however, the RANS model do not agree well with the experimental data compared to the VLES and LES at $\mathrm{x} / \mathrm{L}=0.5,0.7$ while the $\mathrm{r} / \mathrm{H}>0$. The mean $\mathrm{x}$ velocity predicted by LES agrees well with the experimental data, except for $\mathrm{x} / \mathrm{L}=0.7$. The validation of $\mathrm{rms} \mathrm{x}$ velocity is shown in Fig. 4 for the same location and mesh; the rms x velocity profiles are captured well by VLES, LES, and RANS. The rms velocity profiles obtained by VLES and LES exhibit the same trend as the experimental data. The rms velocity predicted by RANS model show better agreement at $\mathrm{x} / \mathrm{L}=0.1$. But the RANS models can't capture the rms velocity at downstream. It appears that the RANS models are unable to capture the rms velocity profile at a region behind the rod due to the flow is disturbed strongly by the rod, whereas the VLES and LES predictions show better agreement. The mean and rms velocity 

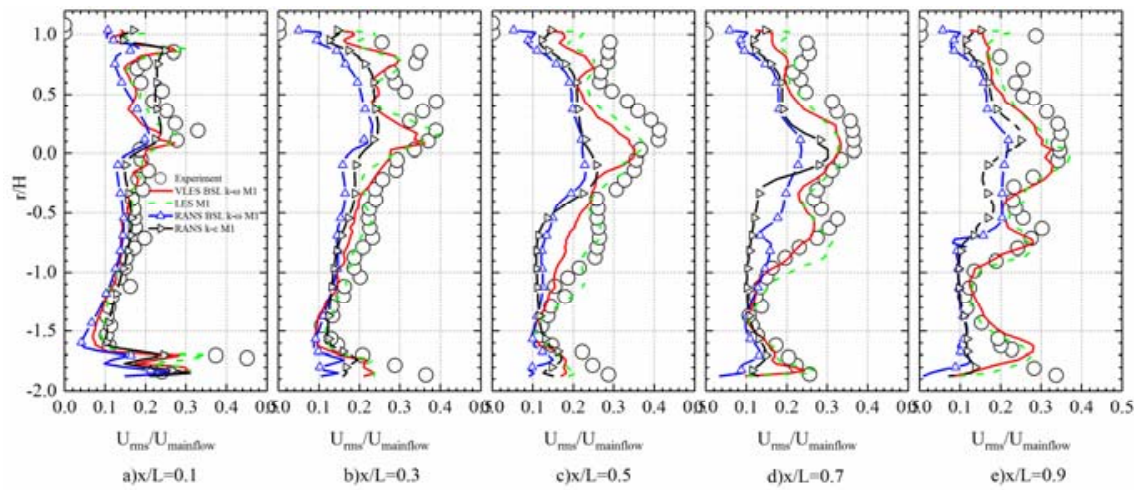

Fig. 4. Radial distributions of $x$ rms velocity(Urms) at plane crossing rods, comparison of experimental data, VLES BSL k- $\omega$ model, LES model, RANS BSL k- $\omega$ model, RANS k- $\varepsilon$ model, on grid M1.

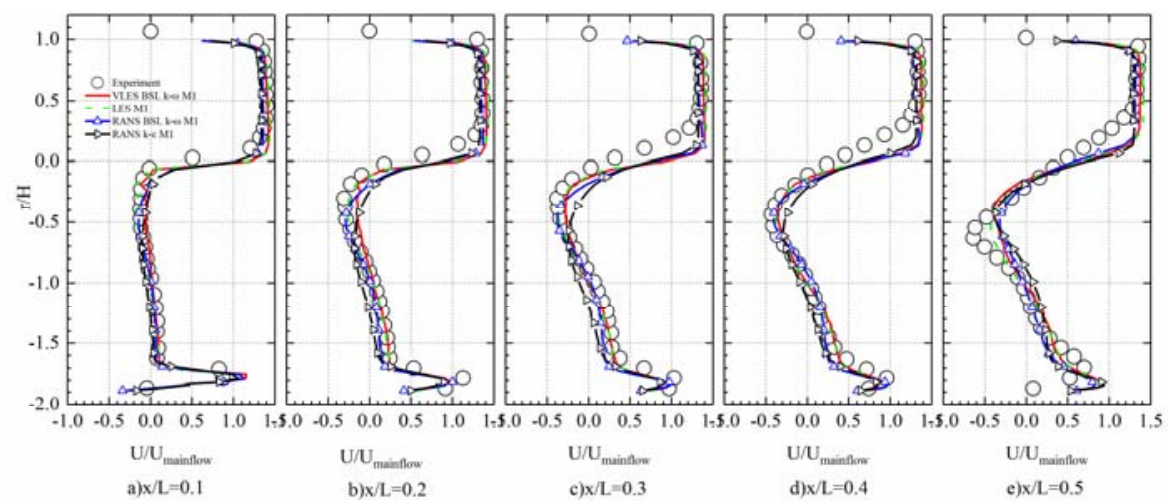

Fig. 5. Radial distributions of $x$ mean velocity $(U)$ at plane between rods, comparison of experimental

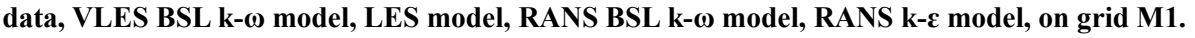

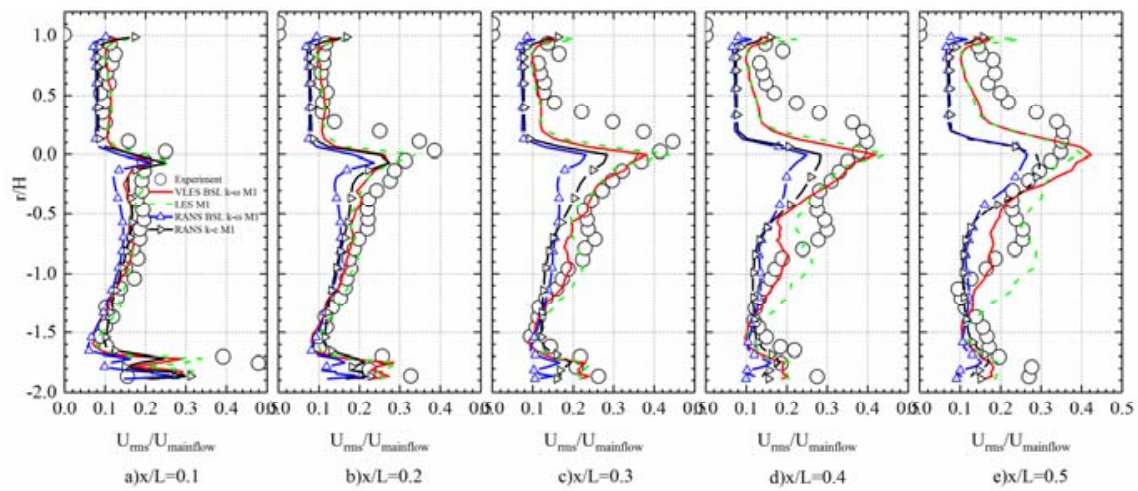

Fig. 6. Radial distributions of $x$ rms velocity(Urms) at plane between rods, comparison of experimental

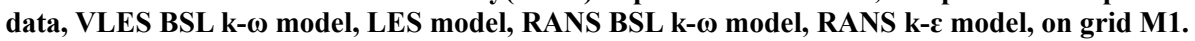

profiles for the plane between rods in different $\mathrm{x}-$ directions are shown in Fig. 5 and Fig. 6, respectively. The mean velocity profiles obtained by the VLES, LES, BSL $k-\omega$, and $k-\varepsilon$ models offer satisfactory predictions in the cavity and mainstream regions, as shown in Fig. 5. For rms velocity, improved velocity predictions are present for $\mathrm{x} / \mathrm{L}=$ $0.1,0.2$, and 0.3 , whereas poor results are present for $\mathrm{x} / \mathrm{L}=0.4$ and $\mathrm{x} / \mathrm{L}=0.5$ for the VLES and LES models. The RANS models can't capture the rms velocity at downstream $(\mathrm{x} / \mathrm{L}=0.4,0.5)$. To examine the accuracy and applicability of the present VLES model further, a fine grid resolution is adopted, denoted by M2. Figure 7 shows the mean $\mathrm{x}$ velocity obtained by the VLES, LES, and RANS models at a plane crossing rods on a fine mesh (M2) compared with the experimental results. It can be seen from Fig. 7 that the agreement between the mean velocity predicted by the VLES and LES and experimental profile, while it is obvious that few velocity discrepancies predicted by the BSL $k-\omega$ and $k-\varepsilon$ models downstream of mainstream $(0.3<\mathrm{r} / \mathrm{H}<1)$. 

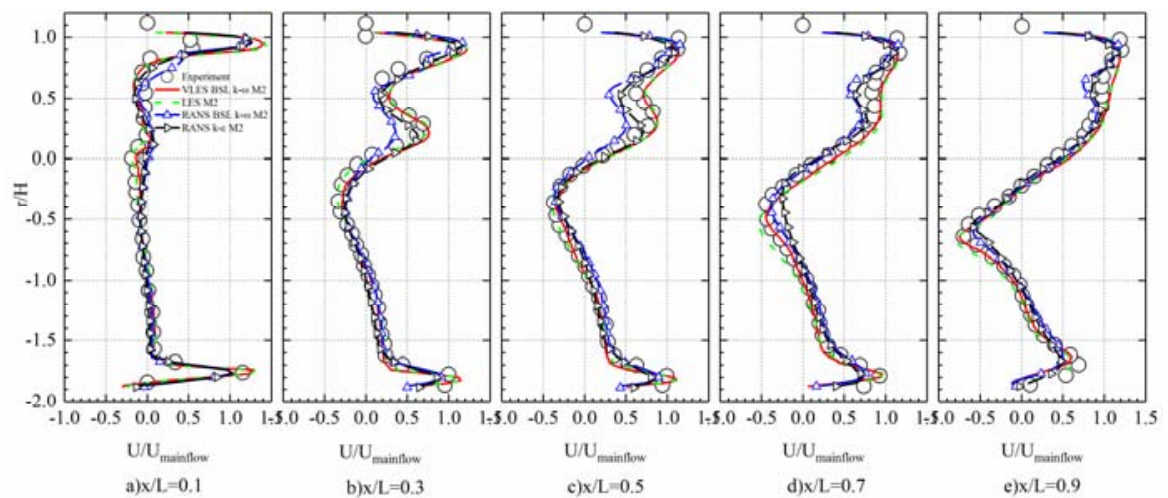

Fig. 7. Radial distributions of $x$ mean velocity $(U)$ at plane crossing rods, comparison of experimental data, VLES BSL k- $\omega$ model, LES model, RANS BSL k- $\omega$ model, RANS k- $\varepsilon$ model, on grid M2.
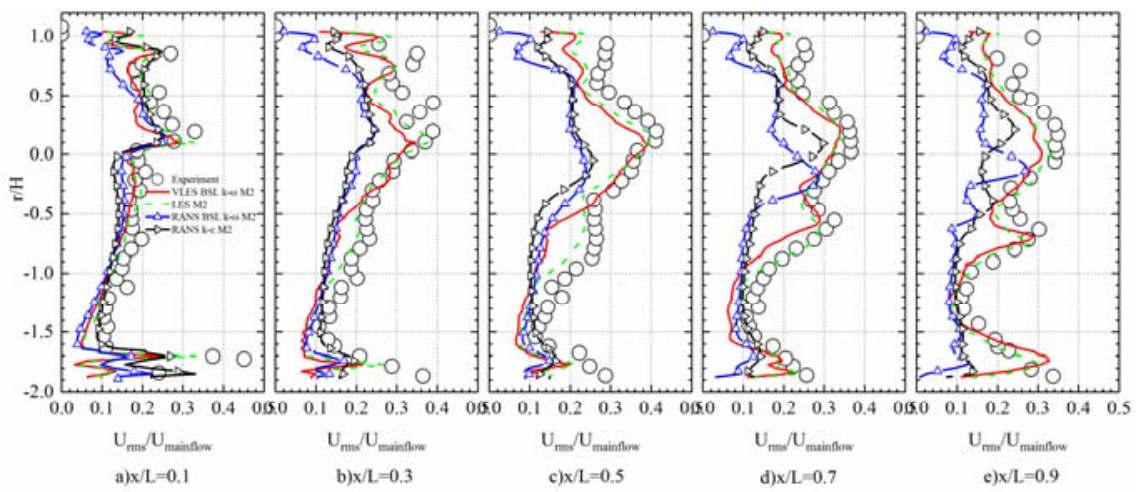

Fig. 8. Radial distributions of $x$ rms velocity $\left(\mathrm{U}_{\mathrm{rms}}\right)$ at plane crossing rods, comparison of experimental

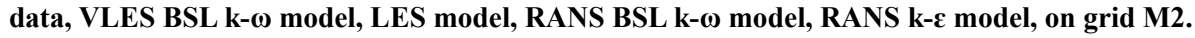
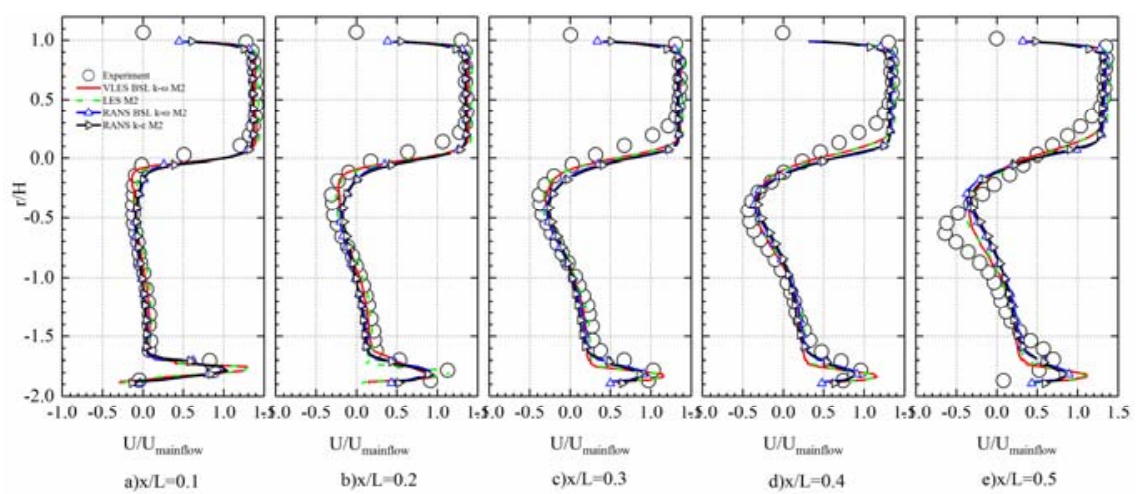

Fig. 9. Radial distributions of $x$ mean velocity $(U)$ at plane between rods, comparison of experimental

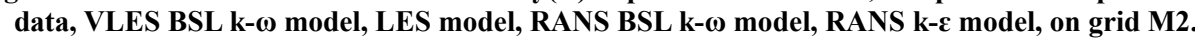

But, it is evident the LES and RANS predictions of M2 are better than those of M1. The evolution trends of rms velocity at the plane crossing rods are all well captured by the VLES and LES models, as shown in Fig. 8.

The mean and rms velocity profiles at the plane between rods on grid M2 are shown in Fig. 9 and Fig. 10. It's evident that all the turbulence models show quite good accuracy in capturing the mean flow velocity. Substantial improvement occurs in the RANS models especially. However, the cells of the
M2 mesh resolution are much significantly more than the cells of M1 resolution. Meanwhile, the mean flow between rods is not as complex as that of flow cross rods, which indicates that for present annular TVC flow the VLES model shows better performance on coarse mesh. For the rms velocity profile at the plane of between rods, there are a number of similarities between $\mathrm{M} 1$ and $\mathrm{M} 2$ at $\mathrm{x} / \mathrm{L}=$ $0.1, \mathrm{x} / \mathrm{L}=0.2$, and $\mathrm{x} / \mathrm{L}=0.3$, as shown in Fig. 10 . The difference between the VLES and LES predictions and experimental measurements is much smaller when using the M2 resolution. 

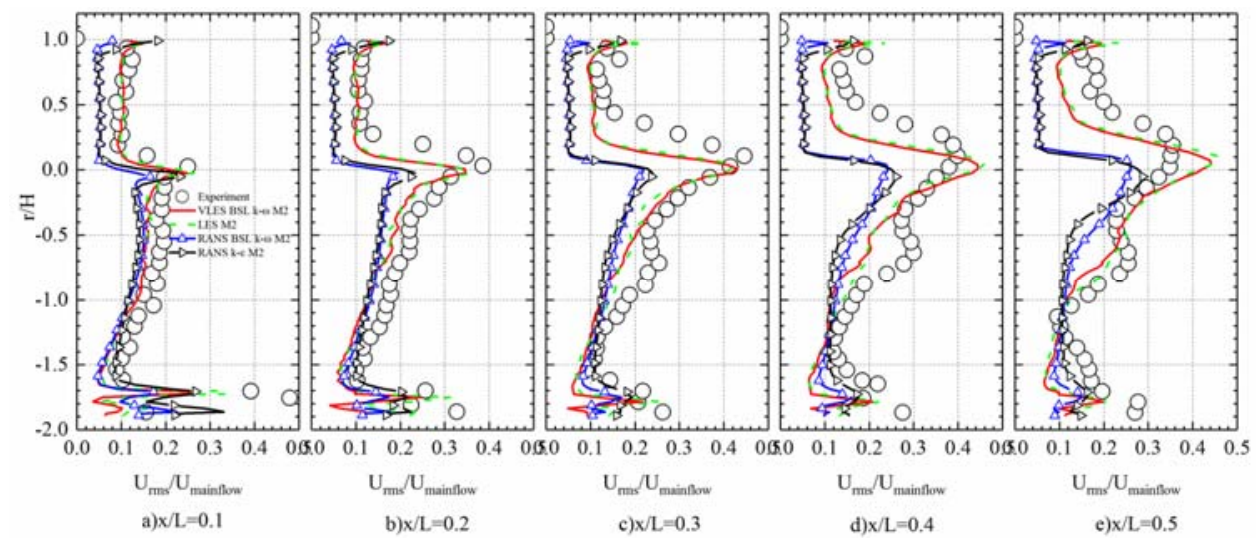

Fig. 10. Radial distributions of $x$ rms velocity(Urms) at plane between rods, comparison of experimental data, VLES BSL k- $\omega$ model, LES model, RANS BSL k- $\omega$ model, RANS k- $\varepsilon$ model, on grid M2.
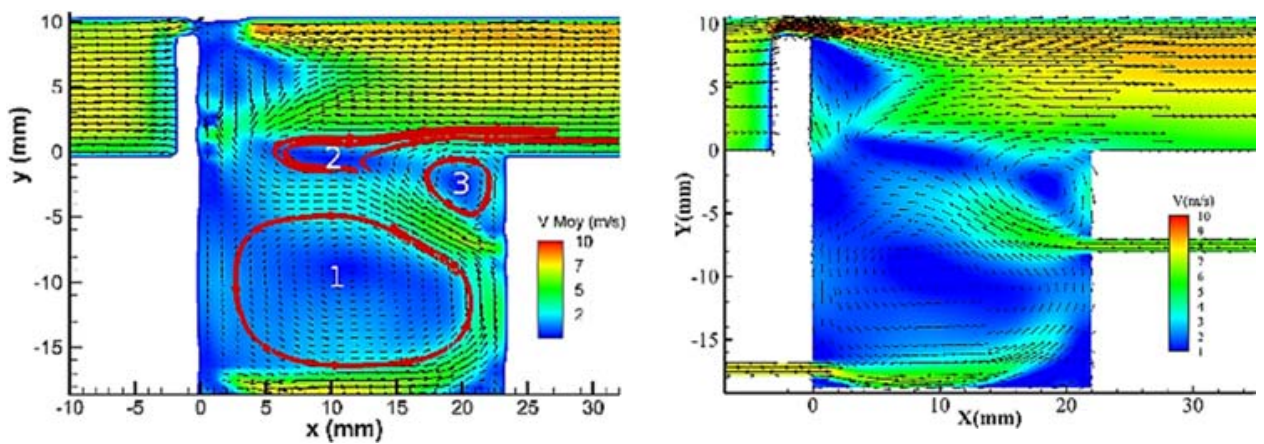

a) Experiment

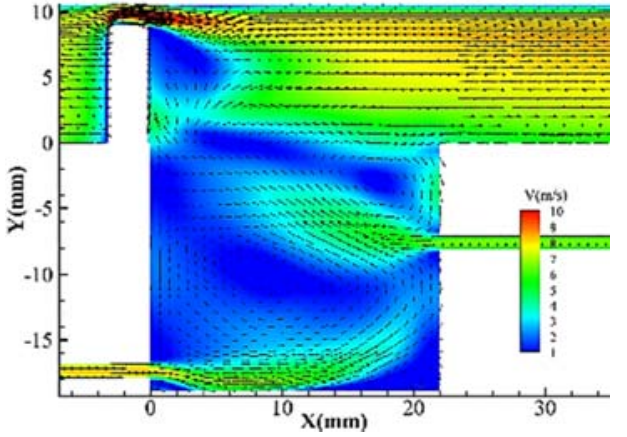

b) VLES BSL k- $\omega$ model

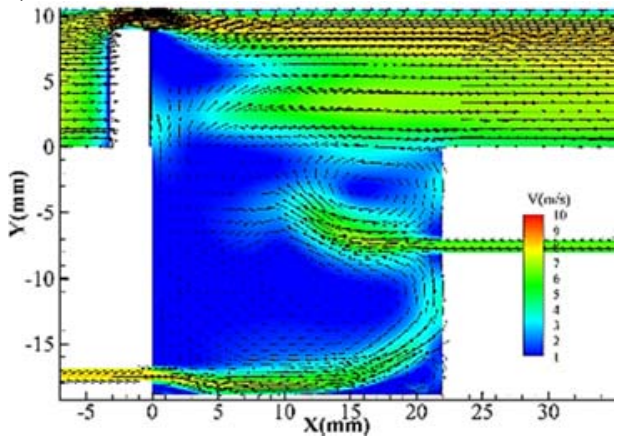

d) RANS BSL k- $\omega$ model

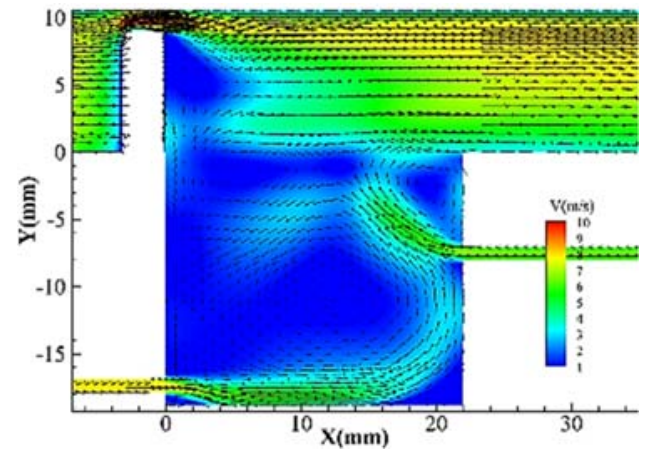

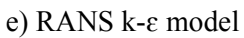

Fig. 11. Contour and vector at plane crossing rods, comparison of experimental data, VLES BSL k- $\omega$ model, LES model, RANS BSL k- $\omega$ model, RANS k- $\varepsilon$ model, on grid M1. 
In conclusion, it can be seen that the better agreement between the VLES BSL $k-\omega$ predictions and the experimental data; meanwhile, the evolution trends of the mean and rms velocity profiles are well captured even using a coarse mesh resolution. However, the two RANS models have relatively good predictive mean velocity but poor predictive rms velocity compared with the experimental data.

\subsection{Flow Pattern and Characteristics}

For a typical combustor, it requires accurate predictions of flow patterns, even small ones. For a numerical method, the natural flow should be accurately predicted first. The mean velocity contour and vectors derived from different models on grids M1 and M2 are shown in Fig. 11- Fig. 14. Vortexes are observed assigned by red lines in the experiment data at the plane crossing rods in Fig. 11 and Fig. 12. And the figures show the velocity contour and vectors at planes crossing/between the rods on grid M1. It is clearly that the flow patterns predicted by the VLES and LES models agree well with the

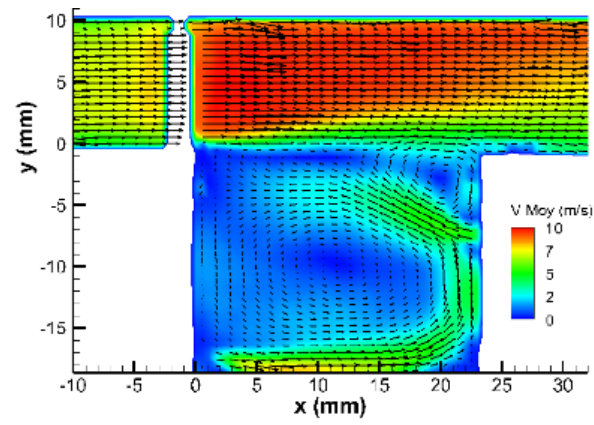

a) Experiment

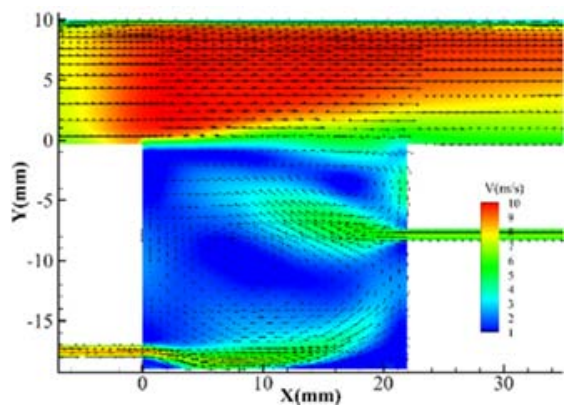

c) LES experimental data. The largest differences exist in the penetration depth of the afterbody air. In the experiment, the penetration depth of the afterbody air is about $0.77 \mathrm{~L}$, which is also observed in the present VLES BSL k- $\omega$ model and LES model. However, the afterbody air penetration depth for RANS BSL $k-\omega$ model and RANS $k-\varepsilon$ model are $0.55 \mathrm{~L}$ and $0.32 \mathrm{~L}$, respectively.

For the fine mesh, Fig. 13 and Fig. 14 show the velocity contours and vectors at the planes behind/between the rods on grid M2. The present VLES BSL $k$ - $\omega$ model and LES model simulation data are in good agreement with the experimental data. Both RANS models show improvement in the penetration depth of the afterbody air as shown in Fig. 13 and Fig. 14. The penetration depth increased to almost $0.77 \mathrm{~L}$. Combining the results from Fig. 11Fig. 14, it is clear that the VLES BSL $k$ - $\omega$ model gives fine flow pattern prediction even based on the coarse mesh resolution.The Q-criterion is employed to visualize the three-dimensional coherent structures in turbulent flows.

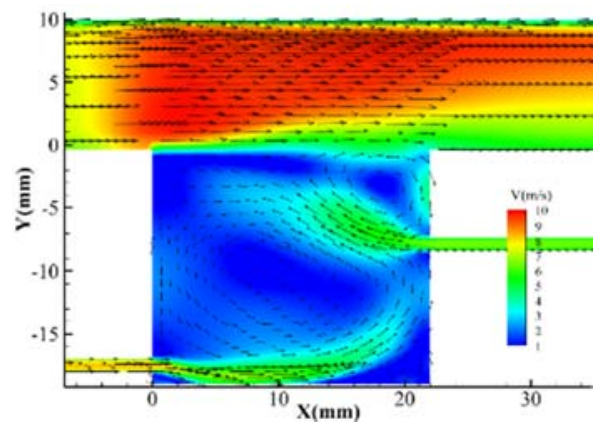

b) VLES BSL k- $\omega$ model

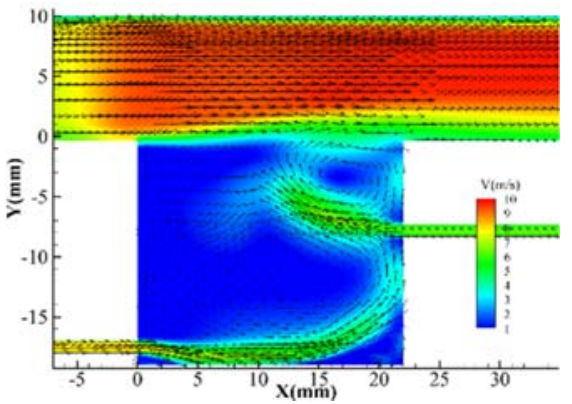

d) RANS BSL k- $\omega$ model

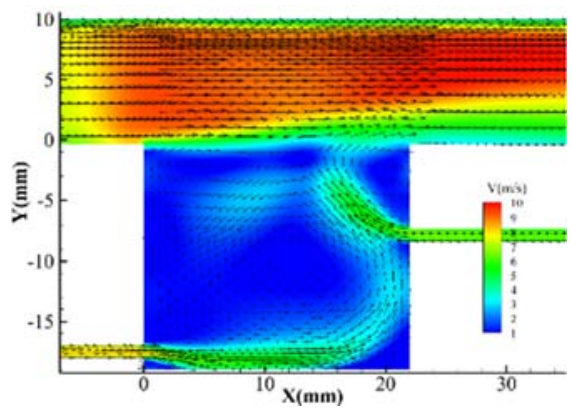

e) RANS k- $\varepsilon$ model

Fig. 12. Contour and vector at plane between rods, comparison of experimental data, VLES BSL k- $\omega$ model, LES model, RANS BSL k- $\omega$ model, RANS k- $\varepsilon$ model, on grid M1. 


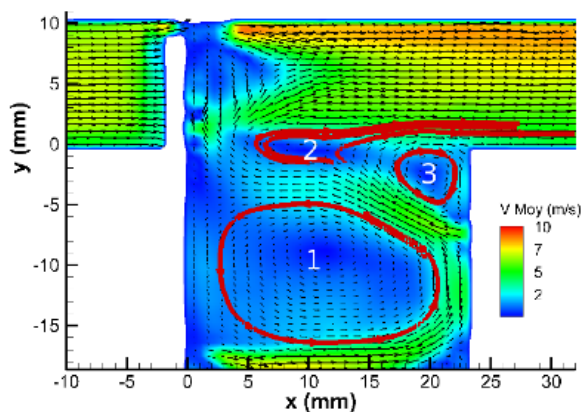

a) Experiment

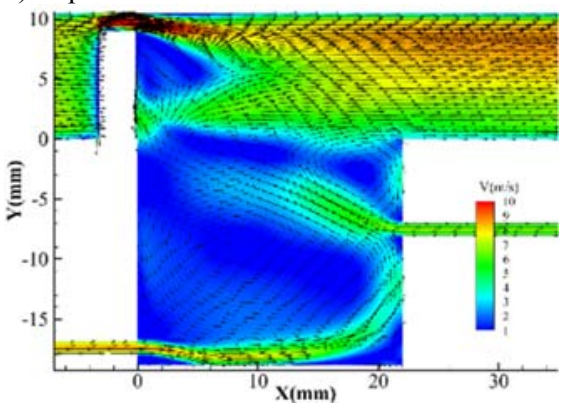

c) LES

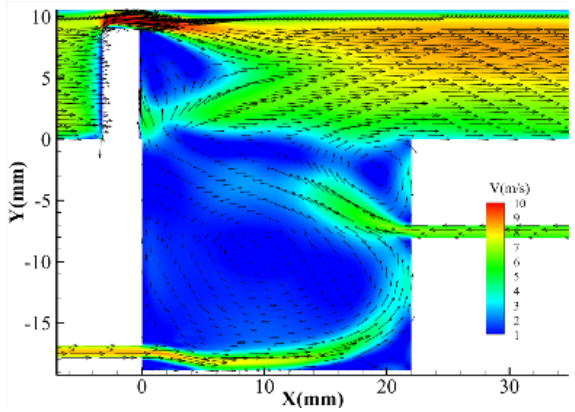

b) VLES BSL k- $\omega$ model

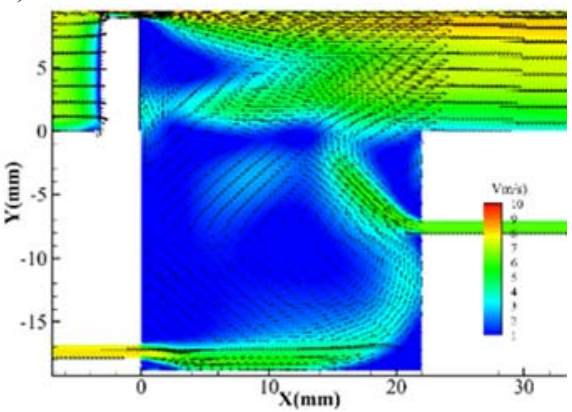

d) RANS BSL k- $\omega$ model

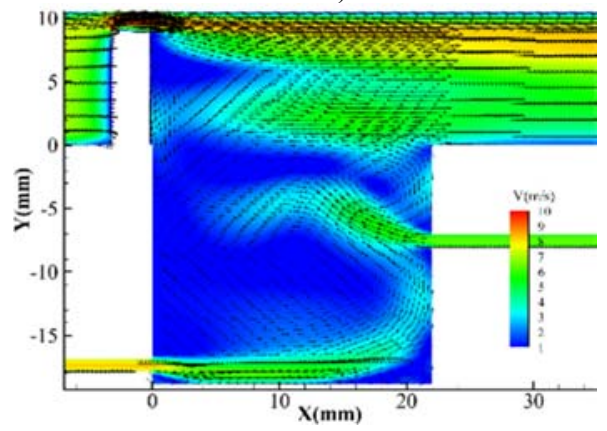

e) RANS k- $\varepsilon$ model

Fig. 13. Contour and vector at plane crossing rods, comparison of experimental data, VLES BSL k- $\omega$ model, LES model, RANS BSL k- $\omega$ model, RANS k- $\varepsilon$ model, on grid M2.

$Q=(1 / 2)\left(\left\|\Omega^{2}\right\|-\left\|S^{2}\right\|\right)$

where $\|\cdot\|$ is the Euclidean matrix norm, $\Omega$ is the symmetric velocity gradient, and $\mathrm{S}$ is the antisymmetric velocity gradient. Figure 15 shows the vortex visualized by the iso-surface of the Qcriterion colored by the mean velocity for the four models on grids M1 and M2. The processing vortex cone (PVC) can be observed in the cavity and mainstream region, especially in the region behind the rods owing to its disturbance to the mainstream flow. The origin vortex cone breaks down into smaller turbulent coherent structures. It is captured by the present VLES BSL $k-\omega$ model and LES model; however, the RANS model fails to capture this. Figure 15 reveals that many more fine vortex structures can be visualized with the gird M2; there is no significant difference between the VLES and LES models. This means that the VLES and LES models both have good accuracy in capturing coherent structures.

Figure 16 shows the contours of the instantaneous turbulent viscosity ratio $\mu_{t} / \mu$ obtained by the present VLES BSL $k-\omega$ model on grids M1 and M2. It should be noted that the maximum value of the turbulent viscosity ratio is 2.0. The parameter in most flow regions is smaller than that in the region behind the rods. It can be seen that the turbulence is modeled mostly in the flow region behind rods owing to the complicated turbulent flow conditions, in which the mainstream air flows past the rods and interacts with the cavity air. It is clear that the ratio becomes smaller in the all flow fields when the mesh solution is refined. Nevertheless, the maximum turbulent viscosity ratio 2 is extremely small on the coarse mesh, which means that the present VLES model resolves most of the turbulence explicitly on such a coarse mesh, and only small amounts of turbulence are modeled. It may contribute to the good velocity predictions, as discussed previously on Fig. 3 to Fig. 
K. Zhang et al. / JAFM, Vol. 15, No. 2, pp. 523-535, 2022.

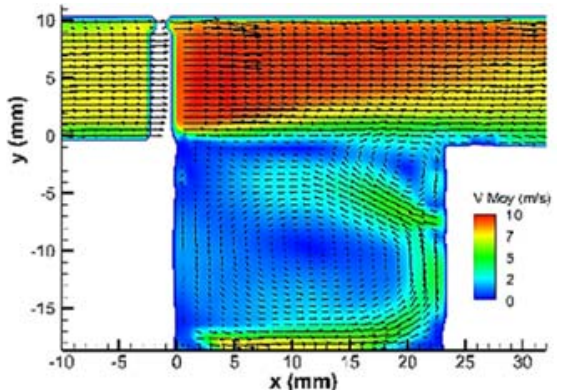

a) Experiment

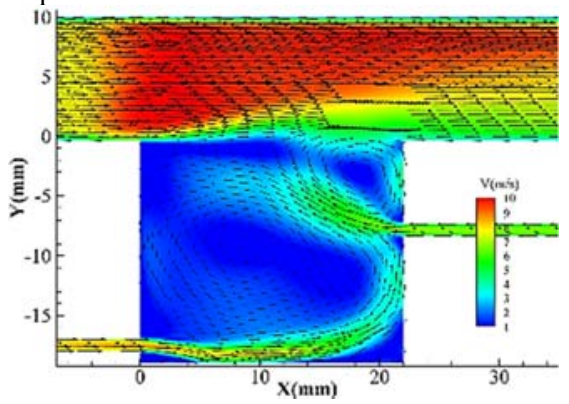

c) LES

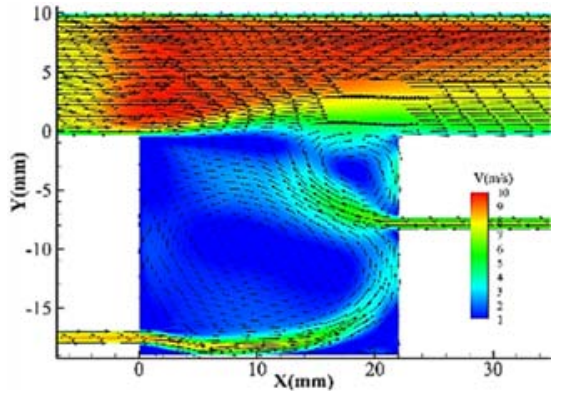

b) VLES BSL k- $\omega$ model

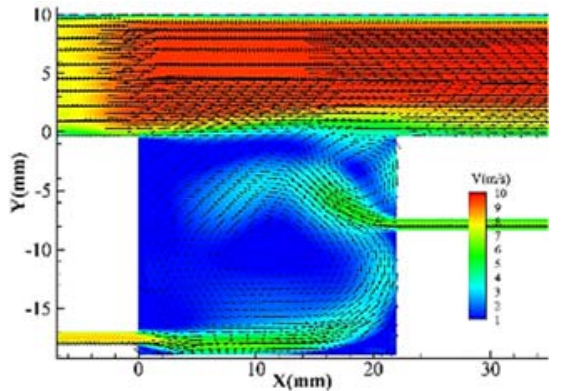

d) RANS BSL k- $\omega$ model

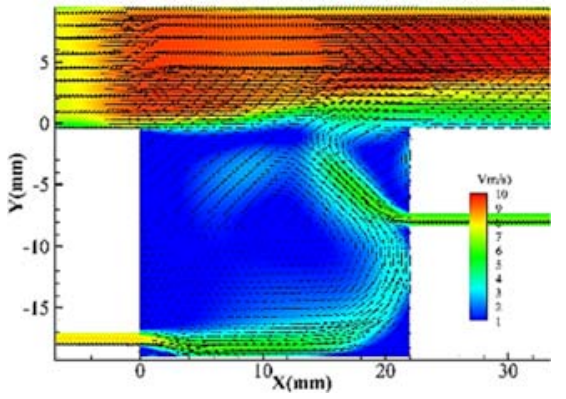

e) RANS k- $\varepsilon$ model

Fig. 14. Contour and vector at plane of between rods, comparison of experimental data, VLES BSL k- $\omega$ model, LES model, RANS BSL k- $\omega$ model, RANS k- $\varepsilon$ model, on grid M2.

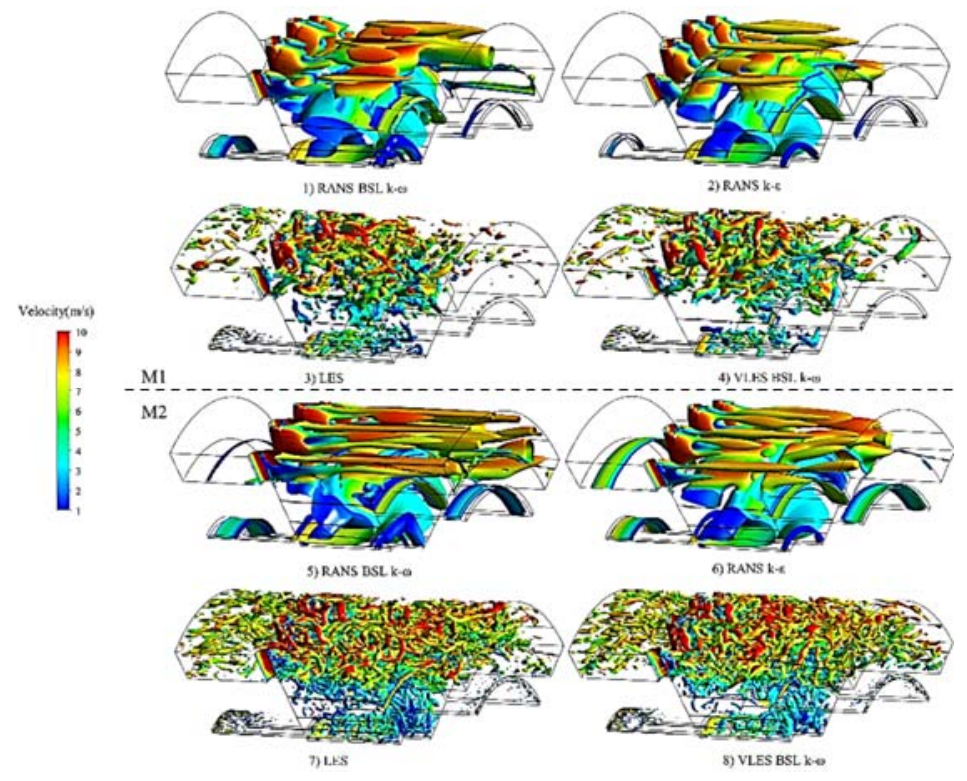

Fig. 15. Coherent vortex visualized by iso-surface of Q-criterion, on grid M1 and M2. VLES BSL k- $\omega$ model and LES model $\left(Q=3.5 E+06 \mathrm{~s}^{-2}\right)$; RANS BSL $\mathrm{k}-\omega$ model and RANS $\mathrm{k}-\varepsilon$ model $\left(\mathrm{Q}=1.6 \mathrm{E}+04 \mathrm{~s}^{-2}\right)$. 


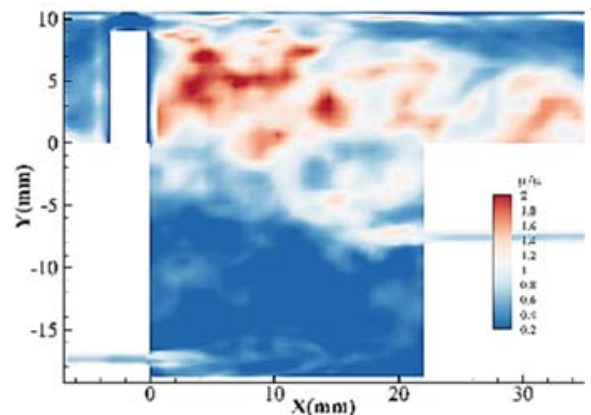

a) plane of behind rods, M1

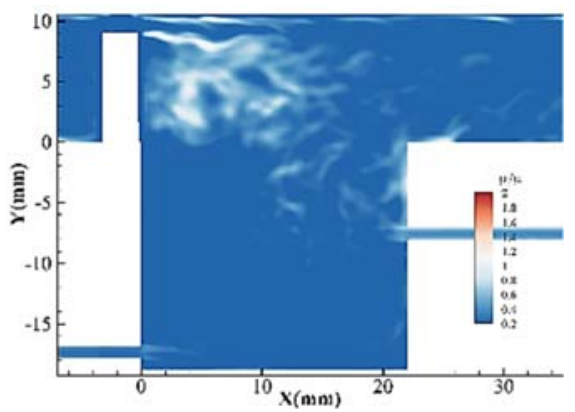

c) plane of behind rods, M2

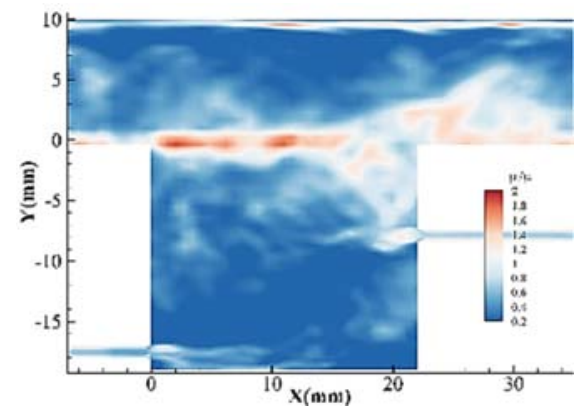

b) plane of between rods, M1

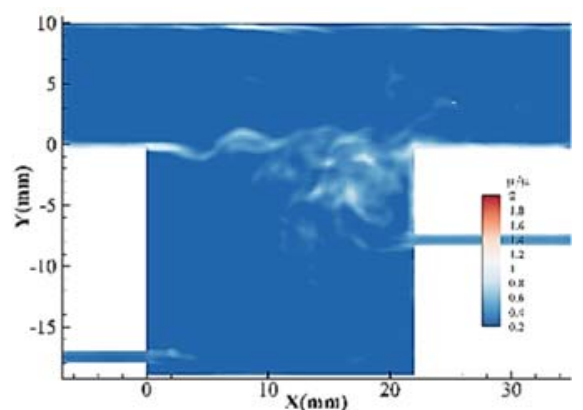

d) plane of between rods, M2

Fig. 16. Contours of the instantaneous turbulent viscosity ratio $\mu \mathrm{t} / \mu$ by the VLES model.

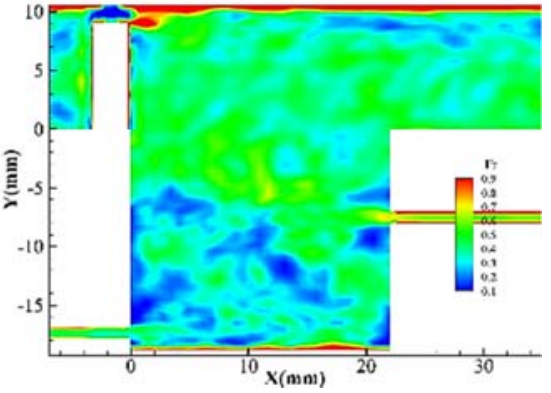

a) plane of behind rods, M1

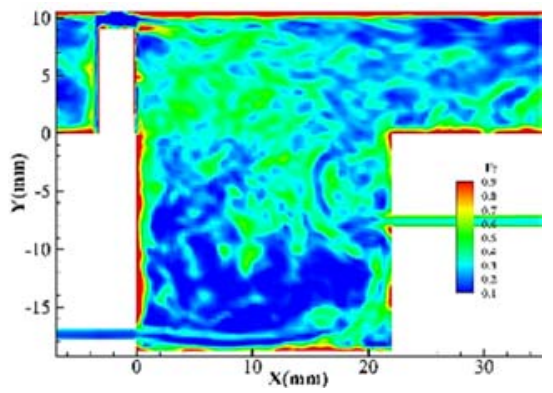

c) plane of behind rods, $\mathrm{M} 2$

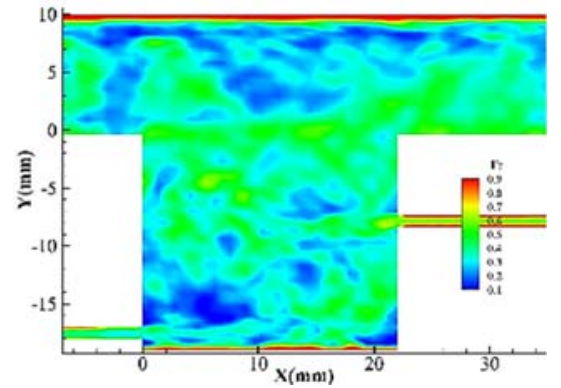

b) plane of between rods, M1

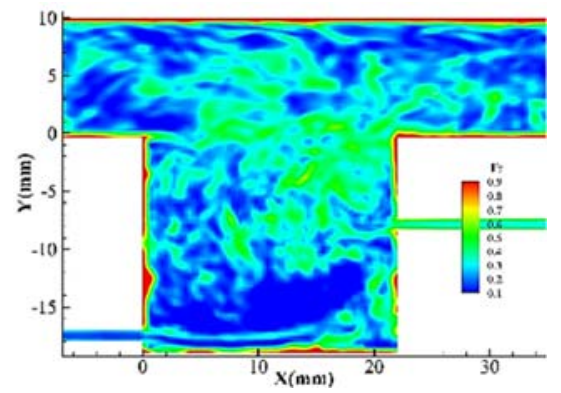

d) plane of between rods, M2

Fig. 17. Contours of the instantaneous Fr by the VLES model.

10. The velocity predictions on the coarse mesh are relatively good also.As discussed above, the parameter $F r$ is the key issue for the VLES model. It has a specific concrete physical meaning, which indicates that the ratio of the unresolved to the total turbulent kinetic energy. The distribution of parameter $\mathrm{Fr}$ is shown in Fig. 17 for the present VLES BSL $k-\omega$ model. The parameter $F r$ approaches 1 in the near-wall region, which means that large amounts of turbulence are modeled and 
RANS modeling is recovered due to the low mesh resolution. However, the $\mathrm{Fr}$ ranges from 0.1 to 0.4 on most of the flow field region far from the wall, and the model behaves like an LES-like method. As for the turbulent viscosity ratio $\mu_{t} / \mu, F r$ is smaller for the fine mesh M2 than for the coarse mesh M1.5.

\section{Conclusions}

In this work, the VLES model is employed to simulate the trapped vortex combustor nonreactive turbulent flow and validate the accuracy of the model. It is implemented in the framework of the BSL $k$ - $\omega$ model, covering the RANS model to the LES model, and finally approaching DNS limit according to the grid resolution. The present VLES BSL $k-\omega$ model provides good velocity prediction for the entire turbulent flow of the trapped vortex combustor when compared with previous experimental data. And the classical vortex structure for trapped vortex combustor is simulated qualitatively by the VLES BSL $k-\omega$ model. The turbulent flow characteristic demonstrates that the present VLES BSL $k-\omega$ model realizes the design aim to transform models. It is found that the new VLES BSL $k$ - $\omega$ model is not sensitive to the gird resolution and provides satisfactory predictions, even on a coarse grid. And the near-wall region grid is will not be treated carefully compared to pure LES. All the results confirm the validity and accuracy of the VLES method and the advantages for application in engineering turbulent flow problems where fine mesh resolution is difficult to generate. The present study confirms the validity and accuracy of the VLES method for annular trapped vortex combustor nonreactive turbulent flow.

What remains to be investigated in future work is the validation of the reacting flow simulation on the combustors.

\section{ACKNOWLEDGEMENTS}

This work was supported by the National Science and Technology Major Project (2017-III-0008-0034).

\section{REFERENCES}

Batten, P., U. Goldberg and S. Chakravarthy (2004). Interfacing Statistical Turbulence Closures with Large-Eddy Simulation. Aiaa Journal, $42,485-492$.

Burguburu, J. (2012). Etude expérimentale de la stabilité d'une flamme dans une chambre de combustion aéronautique par recirculation de gaz brûlés et par ajout d'hydrogène. France.

Chen, S. and D. Zhao (2018). Numerical study of non-reacting flowfields of a swirling trapped vortex ramjet combustor. Aerospace Science and Technology 74, 81-92.

Davidson, L. and S. Dahlström (2005). Hybrid LESRANS: An approach to make LES applicable at high Reynolds number. International
Journal of Computational Fluid Dynamics 19, 415-427.

Fasel, H. F., J. Seidel and S. Wernz (2002). A Methodology for Simulations of Complex Turbulent Flows. Journal of Fluids Engineering 124, 933-942.

Han, X. and S. Krajnović (2012a). An efficient very large eddy simulation model for simulation of turbulent flow. International Journal for Numerical Methods in Fluids 71, 1341-1360.

Han, X. and S. Krajnović (2013a). Validation of a novel very large eddy simulation method for simulation of turbulent separated flow. International Journal for Numerical Methods in Fluids 73, 436-461.

Han, X. and S. Krajnović (2015). Very-Large-Eddy Simulation Based on k- $\omega$ Model. Aiaa Journal 53, 1103-1108.

Han, X. and S. Krajnović (2013b). Very Large Eddy Simulation of Passive Drag Control for a DShaped Cylinder. Journal of Fluids Engineering 135.

Han, X., T. Ye and Y. Chen (2012b). Calibration of a new very large eddy simulation (VLES) methodology for turbulent flow simulation. Science China Physics, Mechanics and Astronomy 55, 1905-1914.

Hsieh, K.-J., F.-S. Lien and E. Yee (2009). Towards a Unified Turbulence Simulation Approach for Wall-Bounded Flows. Flow, Turbulence and Combustion 84, 193.

Hsu, K., L. Gross, D. Trump and W. Roquemore (1995). Performance of a trapped-vortex combustor. In 33rd Aerospace Sciences Meeting and Exhibit: American Institute of Aeronautics and Astronautics.

Jin, Y., X. He, B. Jiang, Z. Wu, G. Ding and Z. Zhu (2014a). Effect of cavity-injector/radial-strut relative position on performance of a trapped vortex combustor. Aerospace Science and Technology 32, 10-18.

Jin, Y., X. He, J. Zhang, B. Jiang and Z. Wu (2014b). Numerical investigation on flow structures of a laboratory-scale trapped vortex combustor. Applied Thermal Engineering 66, 318-327.

Labois, M. and D. Lakehal (2011). Very-Large Eddy Simulation (V-LES) of the flow across a tube bundle. Nuclear Engineering and Design 241, 2075-2085.

Langhe, C. D., B. Merci and E. Dick (2005). Hybrid RANS/LES modelling with an approximate renormalization group. I: Model development. Journal of Turbulence 6, N13.

Liu, N.-S. and T.-H. Shih (2006). Turbulence Modeling for Very Large-Eddy Simulation. Aiaa Journal 44, 687-697.

Menter, F. (2018). Stress-Blended Eddy Simulation (SBES) - A New Paradigm in Hybrid RANS- 
K. Zhang et al. / JAFM, Vol. 15, No. 2, pp. 523-535, 2022.

LES Modeling. In (pp. 27-37). Cham: Springer International Publishing.

Merlin, C., P. Domingo and L. Vervisch (2012). Large Eddy Simulation of turbulent flames in a Trapped Vortex Combustor (TVC) - A flamelet presumed-pdf closure preserving laminar flame speed. Comptes Rendus Mécanique 340, 917-932.

Tiwari, P., Z. Xia and X.Han. (2020). Comparison of VLES and LES Turbulence Modeling for Swirling Turbulent FLow. Journal of Applied Fluid Mechanics 13, 1107-1116.

Sagaut, P. (2006). Large Eddy Simulation for Incompressible Flows (3rd ed.). Berlin: Springer, Berlin, Heidelberg.

Sagaut, P., S. Deck and M. Terracol (2012). Multiscale and Multiresolution Approaches in Turbulence (2nd ed.): IMPERIAL COLLEGE PRESS.

Sharifzadeh, R. and A. Afshari (2020). Numerical investigation of flow field effects on fuel-air mixing in a non-reacting trapped vortex combustor with different injection arrangements. European Journal of Mechanics - B/Fluids 82, 106-122.
Spalart, P. R. (2008). Detached-Eddy Simulation. Annual Review of Fluid Mechanics 41, 181202.

Speziale, C. G. (1998). Turbulence modeling for time-dependent RANS and VLES: A review. Aiaa Journal 36, 173-184.

Xia, Y., P. Sharkey, S. Orsino, M. Kuron, F. Menter, I. Verma, R. Malecki and B. Sen (2021). Stress-Blended Eddy Simulation/Flamelet Generated Manifold Simulation of FilmCooled Surface Heat Transfer and Near-Wall Reaction. Journal of Turbomachinery 143(1).

Xia, Z., X. Han and J. Mao (2020). Assessment and Validation of Very-Large-Eddy Simulation Turbulence Modeling for Strongly Swirling Turbulent Flow. Aiaa Journal 58, 148-163.

Zhang, H., C. Bachman and H. Fasel (2000). Application of a new methodology for simulations of complex turbulent flows. In Fluids 2000 Conference and Exhibit (Vol. 148): American Institute of Aeronautics and Astronautics. 